\title{
Gall stone pulverisation strategy in patients treated with extracorporeal lithotripsy and follow up results of maintenance treatment with ursodeoxycholic acid
}

\author{
M Boscaini, M Piccinni-Leopardi, F Andreotti, A Montori
}

\begin{abstract}
Between November 1988 and July 1992 70 patients with radiolucent gall stones were treated with extracorporeal lithotripsy (ESL) and ursodeoxycholic acid (UDCA; mean (SD) dose $11.2(1.9) \mathrm{mg} / \mathrm{kg} /$ day). Fifty three patients have been followed for one year. One week after lithotripsy, 30.6\% had completely eliminated all stone fragments from the gall bladder and one year later $\mathbf{9 3 . 9 \%}$ were free of stones. Three factors were considered important in achieving these results. 'Pulverisation' of the stone - that is, its fragmentation into echogenic dust (crystalline aggregates, some few hundred $\mu$ in size) or particles similar to grains of sand, smaller than $1 \mathrm{~mm}$ in diameter, or both, is required. Secondly, dust and particles were rapidly eliminated, strongly suggesting a mechanical elimination process by physiological gall bladder contractions. Thirdly, there must be chemical dissolution with biliary acids. This therapeutic approach gave excellent results without causing any clinically relevant side effects. The first 20 patients who became free of stones after ESL were given oral bile acid maintenance treatment $-300 \mathrm{mg} /$ day of UDCA at bedtime, for two years. All were asymptomatic and none had suffered a recurrence after two years. In four patients, crystalline aggregates, detected in gall bladder bile by ultrasound, were subsequently dissolved between one and three months after resuming a full dose regimen of UDCA.

(Gut 1994; 35: 117-121)
\end{abstract}

Results of studies on extracorporeal lithotripsy (ESL) of gall stones in which patients have been followed up for seven years have often proved to be unsatisfactory and at times discordant. Despite this we believe that ESL is an extremely effective method of treating gall stones and that these negative results may result from the following factors that are unrelated to the procedure: (1) Use of equipment developed for kidney stone dissolution and improperly adapted to gall stone treatment; (2) Use of shock waves that are too low in pressure and too few in number; (3) Therapeutic strategy tending towards stone fragmentation followed by chemical dissolution with bile acids lasting even long periods of time.

This trial aimed to determine whether by perfecting the technique of biliary lithotripsy, the procedure would prove to be an effective method of treating gall stones. For this purpose it was necessary to verify:
(1) Whether by using shock waves adequate in pressure and in number it would be possible to achieve 'pulverisation' of stones into crystalline aggregates or particles the size of grains of sand, or both.

(2) Whether by achieving this pulverisation it would be possible to achieve safer and more rapid elimination of the resulting microfragments.

(3) Eliminating stones followed by maintenance treatment while preserving the gall bladder would be sufficient to avoid stone recurrence.

\section{Methods}

PATIENTS

Between November 1988 and July 1992, 70 patients with radiolucent gall stones were treated with a lithotripter equipped with an underwater spark-gap for shock wave generation (Dornier MPL 9000, Germany). The first 53 patients (24 men and 29 women; mean (SD) age 48 (12) (range 24-74 years) and mean (SD) body weight 148.9 (27) (range 108-234) lb) were followed up over one year by monthly clinical and ultrasound examinations. Thirty nine $(73.6 \%)$ were considered to be 'symptomatic' (having had at least one attack of biliary pain lasting more than $\mathbf{3 0}$ minutes in the three years before the treatment), while $14(26 \cdot 4 \%)$ were considered 'asymptomatic' (either having suffered from biliary pain more than three years before treatment or having had an attenuated painful symptomatology). All patients were admitted to hospital: 45 patients $(85 \%)$ for one day and $8(15 \%)$ for two days. No patient required anaesthesia and $17(32 \%)$ required intravenous analgesia (fentanyl $0.1 \mathrm{mg}$ and diazepam $20 \mathrm{mg}$ ). Four of the 53 patients did not complete follow up, leaving only 49 for the full study.

STONES

Thirty four patients had single stones (seven of which had a diameter $>20 \mathrm{~mm}$ ), while 19 had multiple stones (up to eight stones). Five patients had calcified stones: two had a central calcification $2 \mathrm{~mm}$ in diameter, one had a calcified rim $<2 \mathrm{~mm}$, and two had completely calcified stones. The smallest single stone had a diameter of $7 \mathrm{~mm}$ and the largest $40 \mathrm{~mm}$. The mean (SD) stone diameter was $14 \cdot 3(7 \cdot 1 \mathrm{~mm})$. The smallest single stone volume was $0.18 \mathrm{~cm}^{3}$ and the largest was $33.4 \mathrm{~cm}^{3}$. Mean (SD) stone volume was $4 \cdot 4$ (6) $\mathrm{cm}^{3}$. The number and diameter of the stones were measured by ultrasound scan $(3.75 \mathrm{MHz}$ 
convex array probe PVB-358M, SAL 38B Toshiba, Japan). These measurements were determined by oral cholecystography whenever necessary.

\section{ESL TREATMENTS}

Thirty nine patients $(79.6 \%)$ required a single treatment, five required two, two required three, two required four, and one required five for a total of 68 treatments. The mean number of treatments per patient was $1 \cdot 4$, and the percentage that required retreatment was $20 \cdot 4 \%$. The minimum voltage used was $14 \mathrm{kV}$, maximum 23 $\mathrm{kV}$, mean $18 \cdot 8(1) \mathrm{kV}$. The minimum number of shock waves per treatment was 700 , the maximum 2600, and the mean (SD) 1972 (482).

\section{MEDICAL TREATMENT}

Medical treatment consisting of daily doses of ursodeoxycholic acid (UDCA) at bed time (mean (SD) dose $11.2(1.9) \mathrm{mg} / \mathrm{kg} /$ day) started two weeks before ESL treatment and continued for up to three months after patients had been considered 'stone-free'. All patients who were confirmed to be 'stone-free' after three months continued with maintenance therapy (UDCA $300 \mathrm{mg} /$ day at bedtime for up to two years).

\section{ANALYSES}

All patients underwent double contrast oral cholecystography to determine the patency of the cystic duct, ultrasonography of the upper abdomen, plain film $x$ ray of the gall bladder area to detect any stone calcification, chest $x$ ray, electrocardiogram, and urinalysis. In addition, the following blood tests were performed: erythrocyte sedimentation rate, full blood count, prothrombin time, bilirubin, partial thrombin time, aspartate transaminase, alanine amino transferase, lactate dehydrogenase, creatine phosphokinase, gamma glutamyl transferase, alkaline phosphatase, serum amylase and lipase, fasting blood glucose, blood urea nitrogen, creatinine, and pregnancy test in women. Blood tests were repeated the day after treatment. Biochemical variations were considered significant only if they were $20 \%$ or more above or below normal values.

\section{CRITERIA FOR TREATMENT}

Inclusion criteria for treatment were as follows:

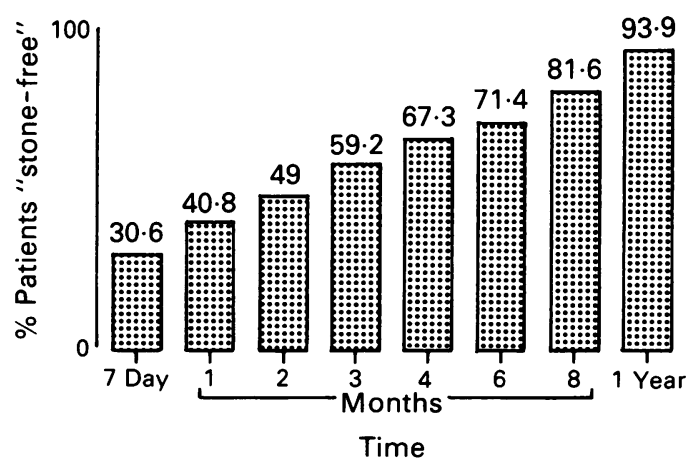

(1) solitary radiolucent stone, maximum diameter $\leqslant 40 \mathrm{~mm}$; (2) multiple radiolucent stones not exceeding $50 \%$ of total gall bladder volume and not exceeding the volume of a stone $40 \mathrm{~mm}$ in diameter; (3) radiolucent stones with rim calcification $<2 \mathrm{~mm}$; (4) radiolucent stones with central calcification $<5 \mathrm{~mm}$; (5) proof of cystic duct patency by oral cholecystography; (6) clear detection of stone by ultrasound and possibility of positioning in shock wave focus.

The exclusion criteria for treatment were as follows: (1) obstructive jaundice; (2) cholangitis; (3) acute cholecystitis; (4) acute pancreatitis; (5) bleeding disorders; (6) cardiac pacemaker; (7) pregnancy.

\section{FRAGMENTATION RESULTS}

Fragmentation results were reported one week after ESL and were defined by ultrasonography by the same operator as follows: (1) Complete fragmentation: echogenic dust (crystalline aggregates, some few hundred $\mu$ in size) or small particles similar to grains of sand with a diameter $<1 \mathrm{~mm}$, or both. The authors call this type of fragmentation 'pulverisation'. (2) Satisfactory fragmentation: remaining particles diameter $\leqslant 5$ mm. (3) Partial fragmentation: remaining particles diameter $>5 \mathrm{~mm}$.

\section{STRATEGY FOR RETREATMENTS}

Reassessment was performed once a month by ultrasonography. If this showed remaining fragments $\leqslant 5 \mathrm{~mm}$, medical treatment was continued with UDCA. Fragments larger than $5 \mathrm{~mm}$ indicated the need for retreatment. This therapeutic approach was followed up to December 1989 (first 25 patients), after which fragments larger than $1 \mathrm{~mm}$ were also retreated at one month intervals in order to achieve pulverisation.

\section{'STONE-FREE' DEFINITION}

Only where there was complete disappearance of stone fragments and also of crystalline aggregates was the patient considered 'stone-free'. This stage had to be confirmed ultrasonographically by the same operator after three months.

\section{STATISTICAL ANALYSES}

To verify whether the attacks of biliary pain suffered after lithotripsy were a side effect of treatment as has been reported,' controls were performed to detect any statistically significant difference between the mean number of attacks suffered after treatment up to the stone-free stage compared with those suffered for an equal period of time before treatment. Statistical analyses were carried out by using paired two tailed Student's $t$ test. A value of $\mathrm{p}<0.05$ was considered to be statistically significant.

\section{Results}

Figure 1 shows the percentage of stone-free patients during the one year follow up period. In all cases stone fragmentation was obtained with 
Figure 2: (A) Sagittal scan before extracorporeal

lithotripsy $(E S L)$. Gall stone of diameter $22 \mathrm{~mm}$ with acoustic shadow. (B)

Sagittal scan immediately after ESL. Stone

'pulverisation': the gall

bladder is completely full of echogenic dust with an echopattern similar to the hepatic parenchima ('solid bile aspect'). (C) Sagittal scan the day after ESL. The gall bladder bile already looks clearer. This scan shows the importance of gall bladder contraction in the process of rapid elimination of microfragments. (D)

Sagittal scan one week after

Sagittal scan one week after
$E S L$ of the same gall bladder which became stone-free.

Residual microfragments or crystalline aggregates can no longer be seen in the gall bladder.
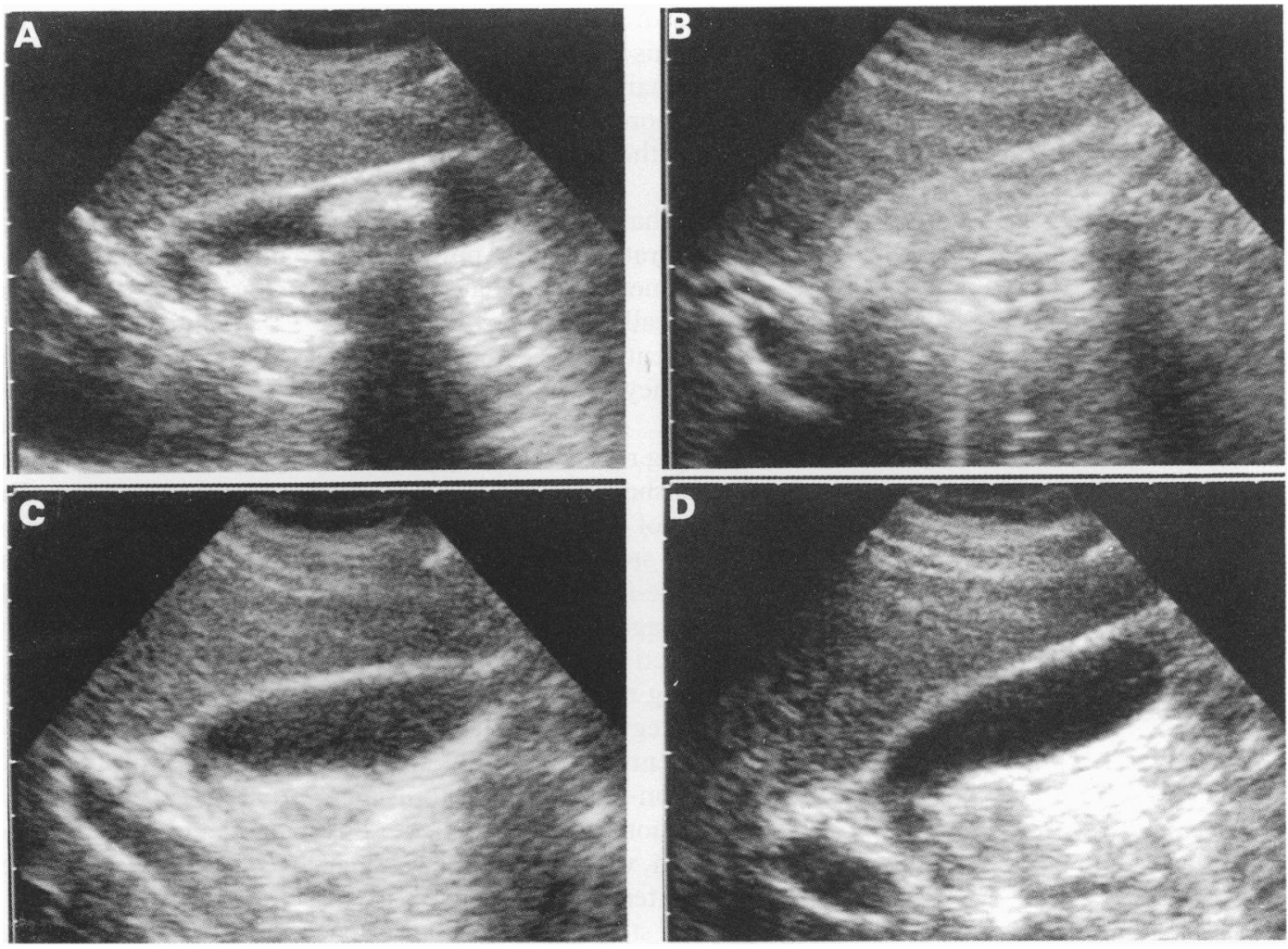

the first treatment: in seven $(14 \cdot 3 \%)$ patients there was partial fragmentation and in seven $(14 \cdot 3 \%)$ satisfactory fragmentation. In 35 $(71 \cdot 4 \%)$ cases complete fragmentation (which we refer to as 'pulverisation') was achieved immediately, $15(42 \cdot 8 \%)$ of these patients were stonefree one week (Fig 2A, B, C, D) and $20(57 \cdot 1 \%)$ one month after treatment. Attacks of biliary pain were not suffered in $24(68.6 \%)$ of the 35 cases of complete fragmentation.

Side effects noticed during treatment were as follows: pain $36.7 \%$; nausea $10 \cdot 2 \%$; and ventricular extrasistoles $2 \%$. After treatment the following side effects were noted: microhaematuria (32.6\%); skin-petechiae (22.4\%); leukocyturia $(16.3 \%)$; increase in total and direct bilirubin (12.2\%); increase in ESR (6\%); albuminuria $(4 \%)$; increase in lactate dehydrogenase $(2 \%)$; increase of creatinine phosphokinase $(2 \%)$.

These abnormalities resolved within a maximum of 10 days after treatment. In six $(12 \cdot 2 \%)$ patients the gall bladder seemed to contract variably from one week up to four months after treatment. These contractions regressed spontaneously, in five cases with 15 to 60 days. In one patient, persistent gall bladder contractions were observed ultrasonographically for seven months, after which the patient failed follow up. In two patients with calcified stones, echogenic foci located in the anterior wall of the gall bladder were detected by ultrasound scan after ESL and produced the 'comet tail artifact'. ${ }^{2}$

As far as reports of biliary pain as a side effect of ESL are concerned, these occurred in $40.8 \%$ of our patients, but we believe that there is no statistically significant difference between the average number of attacks suffered after treatment up to the stone-free stage and the average number of attacks suffered over an equal period of time before treatment. The paired two-tailed Student's $t$ test value comes out 1.75 with a two tail probability level $=0 \cdot 086$. Data show that the difference is highly casual and not therefore significant ( $\mathrm{p}>0.05)$.

All of 20 stone-free patients who were given maintenance therapy of $300 \mathrm{mg} /$ day UDCA at bedtime for two years were asymptomatic, and none suffered a recurrence. In four (20\%) asymptomatic patients crystalline aggregates were seen in the gall bladder $6,9,11$, and 15 months respectively after beginning of maintenance therapy. These crystalline aggregates looked as follows (as previously described by $\left.u^{3}\right)^{3}$ : sedimented dust (three cases) or floating dust (one case). The crystalline aggregates dissolved after one to three months of full dose

\section{Discussion}

There are several important reasons for the unsatisfactory results in published reports to date which help explain the misleading and sometimes unfavourable evaluations of the effectiveness of ESL. One main reason is the use of lithotripters that do not generate the high pressure shock waves that are essential for optimal gall stone fragmentation. In fact, the lithotripters used are often those originally developed for kidney lithiasis and which have been adapted for gall bladder lithiasis. Some of these are also equipped with imprecise ultrasonographic aiming devices that do not allow real-time shooting and consequently risk missing the target which, in the gall bladder, unlike in the kidney, is quite mobile.

Another major reason for the inadequate results has been the improper use of ESL equipment. For fear of potential damage, shock UDCA treatment. 
waves too low in pressure and too few in number have been applied. This concern is unwarrented since we have shown that biliary lithotripsy, with higher pressure and more shock waves, will give excellent results without causing clinically relevant side effects.

A further problem has been the implementation of a therapeutic strategy. Up to now this has aimed at creating fragments which have then had to be dissolved chemically. Because of this it has taken longer for patients to become stone-free and, consequently, they have risked 'residual' symptomatic lithiasis.

The most interesting results, which up to now have never been published, are that $30.6 \%$ of our patients became free of stones after only seven days and $93.9 \%$ were stone-free after one year.

Achieving this stone-free condition in such a short time strongly suggests something new and important - how effective gall bladder contractions are in eliminating microfragments rapidly. The difference in strategy may be substitution of the binomial 'fragmentation-chemical dissolution' for 'pulverisation-mechanical expulsion'. The stone should not be fragmented, but 'pulverised'. This term, which describes the final aim of lithotripsy better, is intended by us to mean reduction of the stone into echogenic dust (crystalline aggregates some few hundred $\mu$ in size) or small particles similar to grains of sand and with a diameter $<1 \mathrm{~mm}$, or both. As has been observed, it is probable that these particles are mainly eliminated by physiological gall bladder contractions. In cases where pulverisation was achieved, the length of time taken to expel particles was extremely short. Some $42 \cdot 8 \%$ patients were stone-free after one week and $57 \cdot 1 \%$ at the end of a month. In $68 \cdot 6 \%$ of the cases elimination occurred without attacks of biliary pain.

Another point we would like to stress is that pulverisation must be achieved in the shortest time possible, even if this requires more treatments at shorter intervals (one month). Reported side effects (mild biliary pancreatitis in $1.8 \%$ of the cases, cholestasis in $1 \%$, and endoscopic sphincterotomy in $0.6 \%)^{1}$ have actually been the result of fragments remaining in the gall bladder for long periods, and thus the consequential residual lithiasis. Damage caused by shock waves has never created any clinically relevant problems except in the rare case of a subcapsular haematoma of the liver in $0 \cdot 1 \%$ of patients.'

A therapeutic strategy aimed at fragment pulverisation should further reduce the risks of common bile duct obstruction and acute biliary pancreatitis. The American National Multicenter Study (MEDSTONE) data indicate that fragments can pass spontaneously or are dissolved, or both, without causing major side effects $^{4}$ and Greiner ${ }^{5}$ has already indicated the feasibility and innocuousness of the passage through the main biliary duct of fragments up to $3 \mathrm{~mm}$ in size.

We emphasise that to obtain stone pulverisation, underwater spark-gap lithotripters able to produce high pressure shock waves and equipped with precision ecographic aiming devices allowing real-time shooting are essential. In addition, an adequate number of shock waves must be applied. While published reports show that this number is usually limited to 1500 on spark-gap equipment, we used a mean (SD) of 1972 (482) waves, up to a maximum of 2600.

As far as side effects are concerned, we show that biliary colic cannot be considered as such, since in our patients there was not a significant difference between the number of episodes of colic suffered before and after treatment.

Having proved that lithotripsy is valid in freeing the gall bladder from stones, we still need to determine whether it is methodologically correct to use this therapeutic technique in patients with a gall bladder that is susceptible to stone recurrences. Ruppin ${ }^{6}$ and Toulet $^{7}$ overestimated the percentage of recurrences, according to Lanzini, ${ }^{8}$ because patients were followed up exclusively by cholecystography and the results were not statistically correct. In the past few years, reports of recurrence after chemical dissolution are in agreement. Each year, the incidence of recurrences is about $10 \%,{ }^{8-13}$ but the risk falls after the first two and a half years ${ }^{14}$ and does not seem to persist after five years. ${ }^{813}$ After ESL and with ultrasound control the risk of recurrence seems to be reduced by about half, as the rate becomes 5\% annually. ${ }^{15}$ According to data on recurrence after bile acid treatment, approximately $50 \%$ of patients remain stone-free after 10 years ${ }^{13}$ and according to data after ESL, approximately $80 \%$ of patients should remain stone-free after four years. ${ }^{15}$ In these patients, the aetiopathogenetic defect responsible for the formation of calculi seems to be transitory only, and cholcystectomy in these cases would be an unnecessary as well as a drastic means of preventing stone recurrences. Hence, ESL together with UDCA treatment seems to be appropriate. This is further confirmed by results published in the past few years on the efficacy of maintenance therapy with UDCA in preventing recurrence. Cases where low doses of CDCA $(375 \mathrm{mg} / \text { day })^{10}$ or UDCA $(3 \mathrm{mg} / \mathrm{kg} / \text { day })^{16}$ were given along with follow up consisting exclusively of cholecystography or cholecystography plus ultrasound show no reduction in the percentage of recurrences. Other more recent studies show that the risk of recurrence is reduced by using higher doses of UDCA during maintenance therapy..$^{11}$

We would like to add to these results our preliminary data on the absence of lithiasic recurrences at two years in patients given maintenance therapy with UDCA $(300 \mathrm{mg} /$ day at bedtime). We believe that these successful results are largely due to the use of precise parameters in ultrasound determination of when a patient can be considered stone-free. If this stage is not evaluated correctly, new lithiasic formations will be inaccurately diagnosed as recurrences whereas, in fact, they represent none other than the volumetric increase of residual microformations (risk of overestimating recurrences). Moreover, if the stone-free condition is not assessed under very rigorous criteria, crystalline aggregates that were not completely eliminated and were not recognised by ultrasonography could become responsible for an accelerated nucleation process (risk of more frequent and earlier recurrences).

Ultrasonographic recognition of crystals in 
Figure 3: (A) Sagittal scan eight days after extracorporeal lithotripsy. Two echogenic images (arrows) projecting a weak acoustic shadow are still present in the gall bladder. Fragments or crystalline aggregates? (B) Sagittal scan after the 'shattering manoeuvre'. The two echogenic images turned into a fine echogenic dust (arrows) proving the presence of crystalline aggregates in the gall bladder.
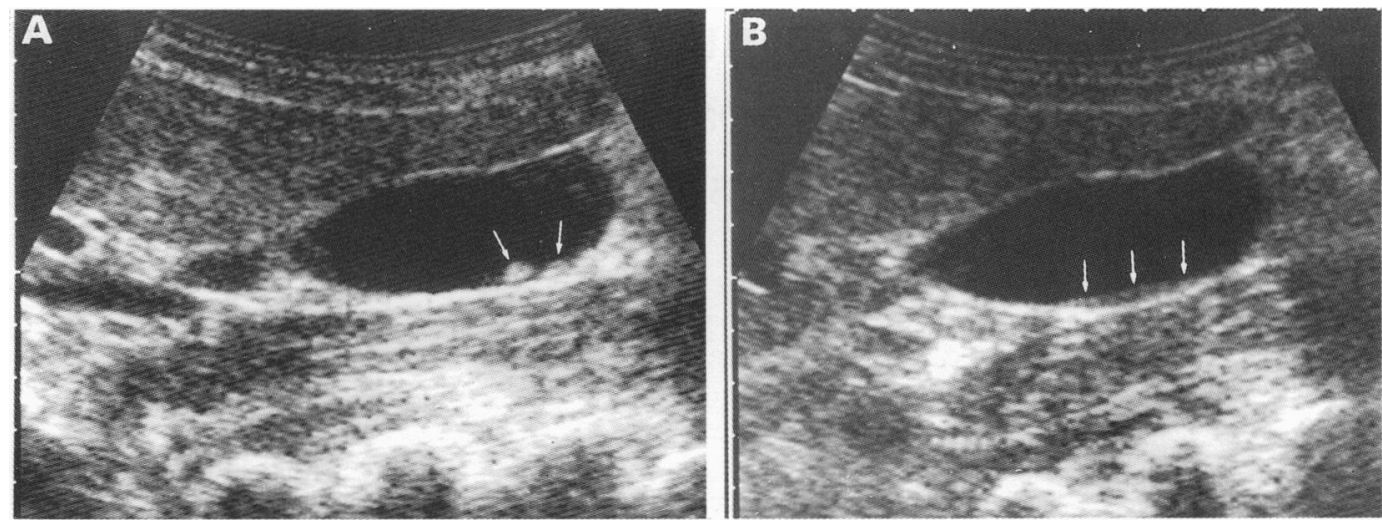

gall bladder bile is an extremely delicate diagnostic requirement with regards to preventing recurrences after reaching the stone-free stage. If the presence of crystals is not recognised early on, the process which we call 'ultrasonic nucleation' and upon which stone formation is based, may not be detected. Ultrasonography, as we have previously shown, allows great diagnostic capabilities in visualising crystalline aggregates in bile which may have multiple, and sometimes quite bizarre, morphological aspects. ${ }^{37}$ We have previously described an interesting aspect of these crystals - 'reversible aggregation' - by which they can simulate the presence of a solid particle sometimes projecting a weak posterior acoustic shadow. We have suggested a 'shattering manoeuvre' ${ }^{3}$ for the differential diagnosis of the echogenic particles contained in bile or adhering to the gall bladder wall, which consists in repeated percussion of the patient's right hypochondrium with the ultrasound probe, without removing it from the skin. If a particle is made of crystalline aggregates, it will probably dissolve into fine echogenic dust. This manoeuvre has proved useful even in follow up of patients treated with ESL in that it has allowed discrimination between residual fragments and false ones made of reversible aggregations of crystalling particles, thus leading to a more correct treatment strategy (Fig 3A, B).

In conclusion, we maintain that the use of equipment suitable for the treatment of biliary lithotripsy, when coupled with the use of adequate power and optimal number of shock waves, will allow the reduction of the stone into echogenic dust consisting of crystalline aggregates or particles similar to grains of sand or both. The fact that these microfragments are quickly eliminated strongly suggest that it is caused by mechanical expulsion through physiological gall bladder contractions. This suggestion can only be confirmed conclusively through additional motility studies without the use of bile acids. An adjuvant medical therapy with UDCA will be necessary to help the elimination process through chemical dissolution of these microfragments. ${ }^{18}$ Recurrences can probably be avoided by giving patients - especially those at high risk follow up maintenance treatment with UDCA and accurate ultrasonographic controls in order to diagnose rapidly the presence of crystals in bile.
We are indebted to Ms Bonnie Bates, Ms Anna Maria Eriksson and Mr Alexander Guttieres, for assistance in preparing the manuscript.

1 Sackmann M, Pauletzki J, Sauerbruch T, Holl J, Schelling G, Paumgartner $\mathrm{G}$. The Munich gallbladder lithotripsy study. Results of the first 5 years with 711 patients. Ann Intern Med 1991; 114: 290-6.

2 Shapiro RS, Winsberg F. Comet-tail artifact from cholesterol crystals: observations in the postlithotripsy gallbladder and an in vitro model. Radiology 1990;177: 153-6.

3 Boscaini M, Magnani G, Mandetta S, Montori A. Morphological appearance of low-level echoes in the gallbladder. Interpretation with microscopic biliary analysis and clinical correlation. Surg Endosc 1987; 1 (1): 41-9. Letter Dornier User 1989; 5: 30-7.

4 Burnett D, Ertan A, Jones R, O'Leary JP, Mackie R, Robinson JE, et al. Use of external shock-wave lithotripsy and adjuvant ursodiol for treatment of radiolucent gallstones. A national multicenter study. Dig Dis Sci 1989; 7: 1011-5.

5 Greiner L, Munks C, Heil W, Jakobeit C. Gallbladder stone fragments in feces after biliary extracorporeal shock-wave lithotripsy. Gastroenterology 1990; 98: 1620-4.

6 Ruppin DC, Dowling RH. Is recurrence inevitable after gallstone dissolution by bile acid treatment? Lancet 1982; I (8265): 181-5

7 Toulet J, Rousselet J, Viteau JM, Duchon Y, Pagniez R, Samain $\mathrm{B}$, et al. Récidives et prévention des récidives après dissolution de la lithiase vésiculaire par l'acide chénodéoxycholique chez 22 patients. Gastroenterol Clin Biol 1983; 7: 605-9.

8 Lanzini A, Jazrawi RP, Kupfer RM, Maudgal DP, Joseph AEA, Northfield TC. Gallstone recurrence after medical dissolution: an overestimated threat? $\mathcal{f}$ Hepatol 1986; 3: 241-6.

9 Salen G. Gallstone dissolution therapy with ursodiol. Efficacy and safety. Dig Dis Sci 1989; 34 (12): 39-43S.

10 Marks JW, Lan SP, the Steering Committee, the National Cooperative Gallstone Study Group. Low dose chenodiol to prevent gallstone recurrence after dissolution therapy. Ann Intern Med 1984; 100: 376-81.

11 Tint GS, Salen G, Chazen D. Symptomatic gallstones are likely to reoccur after dissolution with UDCA, but this may be prevented by low-dose UDCA. Gastroenterology 1987; 92: A1787.

12 Villanova N, Bazzoli F, Taroni F, Frabboni R, Mazzella G, Festi $\mathrm{D}$, et al. Gallstone recurrence after successful oral bile acid treatment. Gastroenterology 1989; 97: 726-31.

13 O'Donnell LDJ, Heaton KW. Recurrence and re-recurrence of gallstones after medical dissolution: a long-term followup. Gut 1988; 29: 655-8.

14 Dowling RH, Gleeson DC, Hood KA, British Belgian Gallstone Study Group. Gallstone recurrence in postdissolution management. In: Paumgartner G, Stiehl A, Gerok W, eds. Bile acids and the liver. Lancaster: MTP Press, 1987: 355-67.

15 Sackmann M, Niller H, Ippisch E, Klueppelberg U, Neubrand M, Pauletzki J, et al. Gallstone recurrence after lithotripsy. Gastroenterology 1992; 4: A332.

16 Hood K, Gleeson D, Ruppin DC, Dowling RH, BBGSG. The British Belgian Gallstone Study Group's (BBGSG) postdissolution trial Gut 1987; 28: A1359.

17 Boscaini M, Mandetta S, Magnani G. Aspects morphologiques des échos à faible intensité dans la vésicule biliare: interprétation échographique, signe de la troisiéme moustache et corrélations cliniques. Le fournal Français d'Echographie 1983; I (2): $23-4$.

18 Schoenfield LJ, Berci G, Carnovale RL, Casarella W, Caslowitz P, Chumley D, et al. The effect of ursodiol on the efficacy and safety of extracorporeal shock-wave lithotripsy of gallstones. The Dornier national biliary lithotripsy study. NEngl f Med 1990; 18: 1239-45. 